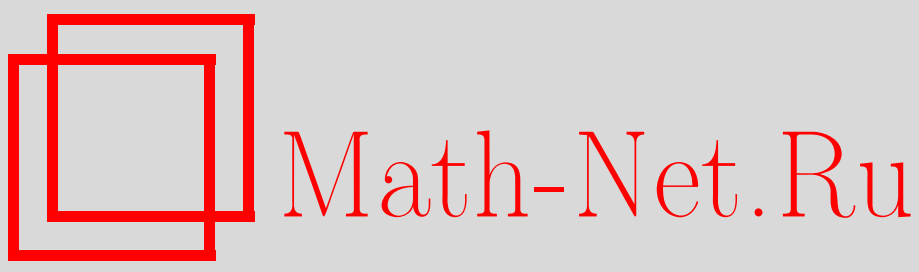

А. А. Толстоногов, Непрерывные селекторы многозначных отображений с невыпуклыми, незамкнутыми, разложимыми значениями, Матем. сб., 1996, том 187, номер 5, 121-142

DOI: https://doi.org/10.4213/sm131

Использование Общероссийского математического портала Math-Net.Ru подразумевает, что вы прочитали и согласны с пользовательским соглашением

http://www.mathnet.ru/rus/agreement

Параметры загрузки:

IP : 34.229 .108 .108

26 апреля 2023 г., 10:49:26 
УДК 517.965

\author{
А. А. Толстоногов
}

\title{
Непрерывные селекторы многозначных отображений с невыпук лыми, незамкнутыми, разложимыми значениями
}

\begin{abstract}
В работе выделяется класс многозначных отображений с невыпукльми, незамкнутыми, разложимыми значениями и доказьваются теоремы существования непрерывных селекторов у таких отображений. К этому классу относятся многозначные отображения, значениями которых являются экстремальные точки непрерьвных многозначных отображений с замкнутьми, выпуклыми, разложимыми значениями в банаховом пространстве интегрируемых по Бохнеру функций. В основу доказательств положена теорема Бэра о категориях. Известно, что в общем случае множество экстремальных точек замкнутого выпуклого множества является незамкнутым. Поэтому результаты этой работы дают ответ на вопрос о существовании непрерьвњых селекторов у многозначных отображений с невьпукльми незамкнутыми значениями.

Библиографоия: 26 названий.
\end{abstract}

Результаты работы [1] явились фундаментальными для изучения проблемы существования непрерывных селекторов у многозначных отображений с невыпукльми, замкнутыми, разложимьми значениями. Идеи этой работы получили свое развитие в [2]-[6] и др. В основу доказательства теорем существования непрерывных селекторов у таких отображений были положены различные обобщения теоремы А.А. Ляпунова о вьпуклости множества значений векторных и многозначных мер. В настоящей работе мы развиваем другой подход, отличный от того, что использовалсяв в [1]-[6] и др. Этот подход базируется на использовании теоремы Бэра о категориях. Следует отметить, что идея использования теоремы Бэра была высказана в [7]. Эта идея развивалась в [8]-[16] и др. для доказательства существования и изучения свойств решений дифференциальных включений. По-видимому, впервые теорема Бэра для доказательства сушествования непрерывных селекторов у многозначных отображений с невыпуклыми, замкнутыми, разложимьми значениями была использована в [5]. В настоящей работе мы продолжаем эти исследования. Представление о содержании работы могут дать следуюшие теоремы.

Пусть $T$ - метризуемый компакт с неотрицательной неатомической мерой Радона $\mu_{0}$ и $\sigma$-алгеброй $\Sigma \mu_{0}$-измеримых подмножеств, $X$ - сепарабельное банахово пространство, $L_{1}(T, X)$ - банахово пространство классов эквивалентности интегрируемых по Бохнеру функций из $T$ в $X, M$-сепарабельное метрическое пространство.

Подмножество $M_{0} \subset M$ называется $\sigma$-компактныц, если оно представимо в виде объединения последовательности компактных множеств.

Пусть $M_{0}$ - плотное в $M \sigma$-компактное множество. В силу сепарабельности $M$ такие множества всегда существуют.

Работа вьполнена при частичной финансовой поддержке Российского фонда фундаментальных исследований (грант № 93-011-264). 
Teорема 0.1. Пусть $\Gamma: M \mapsto L_{1}(T, X)$ - непрерьвное в метрике Хаусдорфа, ограниченное на $M$ многозначное отображение $с$ выпукльми, слабо компактными, разложимыми значениями. Тогда существует непрерывный селектор $g(\cdot)$ отображения $Г$ такой, что

$$
\begin{array}{ll}
g(\xi) \in \operatorname{ext} \Gamma(\xi), & \xi \in M_{0}, \\
g(\xi) \in \overline{\operatorname{ext}} \Gamma(\xi), & \xi \in M \backslash M_{0} .
\end{array}
$$

Если $M$ б-компактно, то включение (0.1) выполняется при всех $\xi \in M$. Здесь ехt $\Gamma(\xi)$ - совокупность всех экстремальных точек множества $\Gamma(\xi)$, а $\overline{\operatorname{ext}} \Gamma(\xi)$ - замыкание в $L_{1}(T, X)$ множества $\operatorname{ext} \Gamma(\xi)$.

Tеорема 0.2. Пусть многозначное отображение $\mathscr{F}: M \mapsto L_{1}(T, X)$ имеет замкнутые, ограниченные, разлоэсимье значения и такое, что отобра-

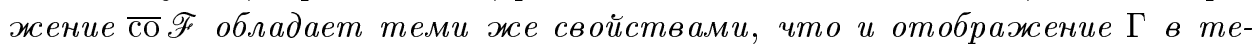
ореме 0.1. Тогда существует непрерывный селектор $f(\cdot)$ отображения Ғ્F такой, что

$$
\begin{aligned}
& f(\xi) \in \operatorname{ext} \overline{\mathrm{co}} \mathscr{F}(\xi), \quad \xi \in M_{0}, \\
& f(\xi) \in \overline{\operatorname{ext}} \overline{\operatorname{co}} \mathscr{F}(\xi), \quad \xi \in M \backslash M_{0} .
\end{aligned}
$$

Если $M$ б-компактно, то включение (0.2) выполняется при всех $\xi \in M$. Здесь $\overline{\mathrm{co}} \mathscr{F}(\xi)$ означает замкнутую выпуклую оболочку множества $\mathscr{F}(\xi)$.

Теорема 0.1 дает условия, при которых многозначное отображение ext $\Gamma$ имеет непрерывный селектор на плотном $\sigma$-компактном множестве $M_{0}$, а теорема $0.2-$ условия, при которых многозначное отображение $\mathscr{F}$, не обладающее какими-либо свойствами непрерывности или полунепрерывности, имеет непрерьвный селектор.

В работе доказываются более обшие результаты, чем приведенные вьше, и рассматриваются примеры отображений $\Gamma$ и $\mathscr{F}$, широко используемых при изучении дифференциальных и эволюционных включений.

1. Пусть $X$ - сепарабельное банахово пространство с нормой $\|\cdot\|, T$ - метризуемьй компакт с положительной неатомической мерой Радона $\mu_{0}$ и $\sigma$-алгеброй $\Sigma \mu_{0}$-измеримых подмножеств, $L_{1}(T, X)$ - банахово пространство классов эквивалентности интегрируемых по Бохнеру функций с нормой

$$
\|v\|_{L_{1}}=\left(\int_{T}\|v(t)\| d \mu_{0}\right) .
$$

Обозначим через $X^{\prime}$ и $L_{1}^{\prime}(T, X)$ пространства, топологически сопряженные к $X$ и $L_{1}(T, X)$, с сильными (нормированными) топологиями. Норму в пространстве $X^{\prime}$ будем обозначать так же, как и в $X$.

Пусть $\left\langle x, x^{\prime}\right\rangle,\left\langle u(\cdot), u^{\prime}(\cdot)\right\rangle$ - канонические билинейные формы, устанавливающие двойственность между $X$ и $X^{\prime}$ и, соответственно, между $L_{1}(T, X)$ и $L_{1}^{\prime}(T, X)$. В дальнейшем, где это не вызывает недоразумений, пространства $L_{1}(T, X)$ и $L_{1}^{\prime}(T, X)$ будем обозначать $L_{1}$ и $L_{1}^{\prime}$.

Пространства $X$ и $L_{1}$ со слабыми $\sigma\left(X, X^{\prime}\right)$ и $\sigma\left(L_{1}, L_{1}^{\prime}\right)$ топологиями [17] будем обозначать $X(\sigma)$ и $L_{1}(\sigma)$. Аналогично, $X^{\prime}(\sigma)$ и $L_{1}^{\prime}(\sigma)$ - пространства $X^{\prime}$ и $L_{1}^{\prime}$ со слабыми $\sigma\left(X^{\prime}, X\right)$ и $\sigma\left(L_{1}^{\prime}, L_{1}\right)$ топологиями. 
Обозначим через $L_{\infty}\left(T, X^{\prime}\right)$ банахово пространство классов эквивалентности скалярно измеримых сушественно ограниченных функций $v: T \rightarrow X^{\prime}$ с нормой

$$
\|v\|_{\infty}=\underset{t \in T}{\operatorname{ess} \sup }\|v(t)\|
$$

В дальнейшем мы не будем различать классы эквивалентности в пространствах $L_{1}$ и $L_{\infty}\left(T, X^{\prime}\right)$ с их представителями.

Известно, что в рамках сделанных предположений на $T$ и $X$ пространства $L_{1}(T, X)$ и $L_{\infty}\left(T, X^{\prime}\right)$ линейно изометричны [18]. Поэтому, как это обычно делается, мы будем считать $L_{\infty}\left(T, X^{\prime}\right)$ сопряженным к $L_{1}(T, X)$ и писать

$$
\left\langle v(\cdot), v^{\prime}(\cdot)\right\rangle=\int_{T}\left\langle v(t), v^{\prime}(t)\right\rangle d \mu_{0}, \quad v(\cdot) \in L_{1},
$$

считая $v^{\prime}(\cdot)$ элементом пространства $L_{\infty}\left(T, X^{\prime}\right)$.

Функция $v: T \rightarrow X^{\prime}$ называется слабо измеримой, если для любого $\varepsilon>0$ сушествует замкнутое множество $T_{\varepsilon} \subset T, \mu_{0}\left(T \backslash T_{\varepsilon}\right)<\varepsilon$, такое, что сужение $v$ на $T_{\varepsilon}$ является непрерывной функцией из $T_{\varepsilon}$ в $X^{\prime}(\sigma)$.

Многозначное отображение $F: T \rightarrow X$ называется измеримым, если для любого замкнутого множества $U \subset X$ множество $t \in T: F(t) \cap U \neq \varnothing$ измеримо.

Многозначное отображение $F: T \rightarrow X$ называется интегрально ограниченнъцм, если существует суммируемая функция $\lambda: T \rightarrow \mathbb{R}^{+}$такая, что

$$
\sup \{\|x\|: x \in F(t)\} \leqslant \lambda(t), \quad t \in T \text {. }
$$

Пусть $Z$ и $Y$ - топологические пространства.

Многозначное отображение $F: Z \rightarrow Y$ называется полунепрерывным снизу в точке $z_{0} \in Z$, если для любого открытого множества $U \subset Y, F\left(z_{0}\right) \cap U \neq \varnothing$, существует окрестность $V\left(z_{0}\right)$ точки $z_{0}$ такая, что $F(z) \cap U \neq \varnothing$ для любого $z \in$ $V\left(z_{0}\right)$.

Многозначное отображение $F: Z \rightarrow Y$ назьвается полунепрерывнылм сверху в точке $z_{0} \in Z$, если для любого открытого множества $U \subset Y, F\left(z_{0}\right) \subset U$, существует окрестность $V\left(z_{0}\right)$ точки $z_{0}$ такая, что $F(z) \subset U$ для любого $z \in V\left(z_{0}\right)$.

Пусть $Y$ - нормированное пространство и $B=\{y \in Y ;\|y\|<1\}$.

Многозначное отображение $F: Z \rightarrow Y$ называется полунепрерывным снизу по Хаусдорфу в точке $z_{0} \in Z$, если для любого $\varepsilon>0$ существует окрестность $V\left(z_{0}\right)$ точки $z_{0}$ такая, что $F\left(z_{0}\right) \subset F(z)+\varepsilon B$.

Многозначное отображение $F: Z \rightarrow Y$ назьвается полунепрерывным сверху nо Хаусдорфу в точке $z_{0} \in Z$, если для любого $\varepsilon>0$ сушествует окрестность $V\left(z_{0}\right)$ точки $z_{0}$ такая, что $F(z) \subset F\left(z_{0}\right)+\varepsilon B$ для любого $z \in V\left(z_{0}\right)$.

Множество $A \subset L_{1}$ назьвается разложсимым, если для любых $u(\cdot), v(\cdot) \in L_{1}$, $E \in \Sigma$ элемент $u(\cdot) \chi_{E}+v(\cdot) \chi_{T \backslash E}$ принадлежит множеству $A$, где $\chi_{E}, \chi_{T \backslash E}{ }^{- \text {ха- }}$ рактеристические функции множеств $E$ и $T \backslash E$. Разложимая оболочка множсесmва $A$ - это наименьшее по включению разложимое множество, содержащее $A$.

Обозначим через $b X$ совокупность всех непустых, ограниченных, замкнутых подмножеств из $X$ с метрикой Хаусдорфа $\operatorname{dist}(\cdot, \cdot)$. Под $c b X(\operatorname{conv} X(\sigma))$ мы понимаем подпространство пространства $b X$, состоящее из выпуклых (выпуклых, 
компактных $)$ подмножеств из $X(X(\sigma))$. Аналогичный смысл имеют пространства $b L_{1}, c b L_{1}$, conv $L_{1}(\sigma)$ с метрикой Хаусдорфа $\operatorname{dist}_{L_{1}}(\cdot, \cdot)$. Пространства $d b L_{1}$, $d c b L_{1}, d$ conv $L_{1}(\sigma)$ представляют собой подпространства пространств $b L_{1}, c b L_{1}$, conv $L_{1}(\sigma)$, состоящие из разложимых множеств.

Обозначим через $\mathscr{M}$ пространство всех числовых мер $\mu: \Sigma \rightarrow \mathbb{R}$ ограниченной вариации, абсолютно непрерывных относительно меры $\mu_{0}$ с нормой $\|\mu\|_{\mathscr{M}}=$ $|\mu|(T)$, где $|\mu|(T)$ - полная вариация меры $\mu$ на $T$.

Пусть $M$ - сепарабельное метрическое пространство и $\mu_{0}(A \triangle B)$ - псевдометрика на $\Sigma$, где $A \triangle B$ означает симметрическую разность множеств $A$ и $B$.

В дальнейшем нам понадобятся утверждения [6].

УТВеРЖДЕНИЕ 1.1. Пусть $\mathscr{P}_{n}: M \rightarrow \mathscr{M}, n \geqslant 1,-$ последовательность полунепрерывных сверху по Хаусдорфу многозначных отображсений с непустыцми относительно компактными значениями, $\left\{V_{n}\right\}_{1}^{\infty}$-локально конечное открытое покрытие пространства $M u\left\{e_{n}\right\}_{1}^{\infty}$ - подчиненное ему непрерывное разбиение единицъ с носителями $\operatorname{supp} e_{n} \subset V_{n}, n \geqslant 1$. Тогда для любого $\varepsilon>0$ существует последовательность непрерывных относительно псевдометрики $\mu_{0}(A \triangle B)$ на $\Sigma$ отображений $\mathscr{B}_{n}: M \rightarrow \Sigma, n \geqslant 1$, такая, что:

1) для каждого $\xi \in M$ множества $\left\{\mathscr{B}_{n}(\xi)\right\}_{1}^{\infty}$ попарно не пересекаются $u \mu_{0}\left(\bigcup_{n=1}^{\infty} \mathscr{B}_{n}(\xi)\right)=\mu_{0}(T)$

2) для любых $\xi \in M, n \geqslant 1$, множество $\mathscr{B}_{n}(\xi)=\varnothing$ тогда и только тогда, когда $e_{n}(\xi)=0$

3) для любых $\xi \in M, n \geqslant 1, \mu(\cdot) \in P_{n}(\xi)$

$$
\left|\mu\left(\mathscr{B}_{n}(\xi)\right)-e_{n}(\xi) \mu(T)\right|<\varepsilon .
$$

Обозначим через $\partial(\|x\|)$ субдифференциал в нуле функции $\|x\|$, который равен $\left\{x^{\prime} \in X^{\prime}:\|x\| \leqslant 1\right\}[19]$.

УТВеРЖДЕНИЕ 1.2. Для любой непрерьвной функции $\gamma: M \rightarrow(0,+\infty)$ существует функиия $v: T \times X \times M \rightarrow X^{\prime}$ со следующими свойствами:

1) для каждыих $(x, \xi) \in X \times M$ функция $v(t, x, \xi)$ слабо измерима по $t$;

2) для почти всех $t$ функиия $v(t, x, \xi)$ непрерьвна по совокупности $(x, \xi)$ в топологии пространства $X^{\prime}(\sigma)$;

3) при почти всех $t \in T$ для каждого $(x, \xi)$

$$
\|x\| \leqslant\langle x, v(t, x, \xi)\rangle+\gamma(\xi), \quad v(t, x, \xi) \in \partial(\|x\|) .
$$

Множество $H \subset L_{1}(T, X)$ называется равномерно интегрируемым, если для любого $\varepsilon>0$ сушествует $\delta>0$ такое, что для любого $E \in \Sigma, \mu_{0}(E) \leqslant \delta$ и любого $f \in H$ выполняется неравенство $\int_{E}\|f(t)\| d \mu_{0}<\varepsilon$.

УТВЕРЖДЕНИЕ 1.3. Пусть $f_{n}, f \in L_{1}(T, X), n \geqslant 1$. Если последовательность $f_{n}, n \geqslant 1$, равномерно интегрируема и $f_{n}(t), n \geqslant 1$, сходится почти всюдуна $T \kappa f(t)$, то

$$
\lim _{n \rightarrow \infty} \int_{T}\left\|f_{n}(t)-f(t)\right\| d \mu_{0}=0
$$


ДоказАтеЛЬСтво. Рассмотрим последовательность числовых функций $z_{n}$, $z_{n}(t)=\left\|f_{n}(t)-f(t)\right\|, n \geqslant 1$, которая равномерно интегрируема и почти всюду сходится к нулю. Из известной теоремы А.А. Ляпунова следует, что множество $\left\{\mu_{0}(E) ; E \in \Sigma\right\}$ является выпуклым и компактным. Следовательно, для произвольного $\delta>0$ существует совокупность $A_{n} \in \Sigma, 1 \leqslant n \leqslant N$, попарно непересекающихся множеств таких, что $T=\sum_{n=1}^{N} A_{n}, \mu_{0}\left(A_{n}\right) \leqslant \delta, 1 \leqslant n \leqslant N$. Отсюда, в силу равномерной интегрируемости последовательности $z_{n}, n \geqslant 1$, следует, что последовательность $z_{n}, n \geqslant 1$, ограничена в $L_{1}(T, R)$. Поэтому она является относительно слабо компактным подмножеством пространства $L_{1}(T, R)$ [18]. Теперь утверждение вытекает из теоремы Данфорда-Петтиса [18].

2. Пусть $\left\{x_{s}^{\prime}\right\}_{1}^{\infty}$ - счетное, плотное в топологии пространства $X^{\prime}(\sigma)$, уравновешенное подмножество множества $\left\{x^{\prime} \in X^{\prime} ;\left\|x^{\prime}\right\| \leqslant 1\right\}$. Для множества $A \in c b X$ обозначим через $\operatorname{ext} A$ совокупность всех его экстремальных точек и определим функции

$$
d^{s}(A)(u)=\sup \left\{\left\langle y-x, x_{s}^{\prime}\right\rangle ; y, x \in A, u=\frac{x+y}{2}\right\}, \quad s \geqslant 1 .
$$

Следуюшая лемма является бесконечномерной версией леммы 1 [15].

\section{Лемма 2.1. Для каждого $s \geqslant 1$}

а) функция $u \rightarrow d^{s}(A)(u)$ неотрицательна, вогнута и если $u \in A \subset B$, $B \in c b X, \operatorname{mo~}^{s}(A)(u) \leqslant d^{s}(B)(u)$;

б) $u \in \operatorname{ext} A$ тогда и только тогда, когда $d^{s}(A)(u)=0$ для всех $s \geqslant 1$;

в) если $Y$ - топологическое пространство, $A: Y \rightarrow c b X$ - непрерывное в метрике Хаусдорфа многозначное отображение и и(у) - его непрерывный селектор, то функиия $y \rightarrow d^{s}(A(y))(u(y))$ полунепрерьвна сверху.

ДокАЗАТЕльство. Доказательство утверждений а), б) мы опускаем, поскольку они с достаточно очевидньми видоизменениями повторяют доказательства аналогичных утверждений в [15]. Следует только учесть, что множество $\left\{x_{s}^{\prime}\right\}_{1}^{\infty}$ разделяет точки пространства $X[17]$.

Остановимся на доказательстве утверждения в). Обозначим через $X^{\prime \prime}$ второе топологически сопряженное к $X$ пространство с нормированной топологией. Будем рассматривать $X$ как подпространство пространства $X^{\prime \prime}$. Тогда $A(y) \subset X^{\prime \prime}$, $y \in Y$, является относительно компактным, выпуклым подмножеством пространства $X^{\prime \prime}$ в слабой топологии $\sigma\left(X^{\prime \prime}, X^{\prime}\right)[17]$. Обозначим через $\bar{A}(y), y \in Y$, замыкание множества $A(y)$ в $\sigma\left(X^{\prime \prime}, X^{\prime}\right)$ топологии. Так как шар $\{x \in X ;\|x\| \leqslant 1\}$ плотен в шаре $\left\{x^{\prime \prime} \in X^{\prime \prime} ;\left\|x^{\prime \prime}\right\| \leqslant 1\right\}$ в $\sigma\left(X^{\prime \prime}, X^{\prime}\right)$ топологии, а топология пространства $X^{\prime \prime}$ индуцирует топологию пространства $X$, то многозначное отображение $\bar{A}(y)$, $y \in Y$, непрерывно в метрике Хаусдорфа $\operatorname{dist}_{X^{\prime \prime}}(\cdot, \cdot)$ на пространстве всех выпуклых, замкнутых, ограниченных подмножеств из $X^{\prime \prime}$.

Из компактности множества $\bar{A}(y), y \in Y$, в $\sigma\left(X^{\prime \prime}, X^{\prime}\right)$ топологии следует, что отображение $\bar{A}(y), y \in Y$, полунепрерывно сверху в $\sigma\left(X^{\prime \prime}, X^{\prime}\right)$ топологии. Тогда многозначное отображение $C(y)=(\bar{A}(y)-u(y)) \cap(-\bar{A}(y)+u(y))$ является полунепрерывным сверху в $\sigma\left(X^{\prime \prime}, X^{\prime}\right)$ топологии как пересечение двух полунепрерывных сверху отображений. 
Легко проверить, что

$$
\begin{aligned}
d^{s}(A(y))(u(y)) & =\sup \left\{\left\langle z-x, x_{s}^{\prime}\right\rangle ; x, z \in \bar{B}(y)-u(y), x+z=0\right\} \\
& =2 \sup \left\{\left\langle 2 z, x_{s}^{\prime}\right\rangle ; z \in C(y)\right\} .
\end{aligned}
$$

Отсюда и из полунепрерьвности сверху $C(y)$ в $\sigma\left(X^{\prime \prime}, X^{\prime}\right)$ топологии следует, что функция $d^{s}(A(y))(u(y))$ полунепрерывна сверху. Лемма доказана.

Для множества $A \in c b X$ обозначим через

$$
g^{s}(A)=\sup \left\{\left\langle x-y, x_{s}^{\prime}\right\rangle ; x, y \in A\right\}, \quad s \geqslant 1 .
$$

Ясно, что $g^{s}(A) \geqslant 0$ и $d^{s}(A)(u) \leqslant g^{s}(A)$ для любого $u \in A$. Пусть

$$
\begin{gathered}
L^{s}(A)(u)=\left\{r \in \mathbb{R}^{+} ; d^{s}(A)(u) \leqslant r \leqslant g^{s}(A)\right\}, \\
A_{n} \in c b X, \quad u_{n} \in A_{n}, \quad n \geqslant 1, \\
A \in c b X, \quad u \in A, \quad r \in L^{s}(A)(u) .
\end{gathered}
$$

Обозначим через $r_{n} \in L^{s}\left(A_{n}\right)\left(u_{n}\right)$ точку такую, что

$$
\left|r-r_{n}\right|=\min \left\{|r-k| ; k \in L^{s}\left(A_{n}\right)\left(u_{n}\right)\right\} .
$$

Лемма 2.2. Пусть последовательность $A_{n} \in c b X, n \geqslant 1$, сходится $\kappa$ $A \in c b X$ в метрике Хаусдорфа, а последовательность $u_{n}, n \geqslant 1$, сходится $к и$. Тогда при фиксированных $s \geqslant 1, r \in L^{s}(A)(u)$ последовательность $r_{n} \in L^{s}\left(A_{n}\right)\left(u_{n}\right), n \geqslant 1$, сходится $\kappa r$.

ДокАЗАТЕЛЬство. Покажем вначале, что последовательность $g^{s}\left(A_{n}\right), n \geqslant 1$, сходится к $g^{s}(A)$. Пусть $\varepsilon>0$ произвольно. Тогда найдется номер $N$ такой, что

$$
A \subset A_{n}+\varepsilon B, \quad A_{n} \subset A+\varepsilon B, \quad n \geqslant N,
$$

где $B=\{x \in X ;\|x\|<1\}$. Возьмем произвольные $x, y \in A$. Из левого включения (2.4) вытекает, что $x=x_{n}+v_{n}, y=y_{n}+w_{n}, n \geqslant N$, где $x_{n}, y_{n} \in A_{n}$, $v_{n}, w_{n} \in \varepsilon B$. Следовательно,

$$
\left\langle x-y, x_{s}^{\prime}\right\rangle=\left\langle x_{n}-y_{n}, x_{s}^{\prime}\right\rangle+\left\langle v_{n}-w_{n}, x_{s}^{\prime}\right\rangle .
$$

Отсюда непосредственно следует

$$
g^{s}\left(A_{n}\right) \leqslant g^{s}(A)+2 \varepsilon, \quad n \geqslant N .
$$

Аналогично, воспользовавшись правым включением (2.4), получим

$$
g^{s}(A) \leqslant g^{s}\left(A_{n}\right)+2 \varepsilon, \quad n \geqslant N .
$$

Тем самьм последовательность $g^{s}\left(A_{n}\right), n \geqslant 1$, сходится к $g^{s}(A)$. Поэтому в соответствии с утверждением в) леммы 2.1 для любого $\varepsilon>0$ найдется номер $N$ такой, что для всех $n \geqslant N$

$$
d^{s}\left(A_{n}\right)\left(u_{n}\right) \leqslant d^{s}(A)(u)+\varepsilon, \quad g^{s}(A) \leqslant g^{s}\left(A_{n}\right)+\varepsilon .
$$

Следовательно,

$$
L^{s}(A)(u) \subset L^{s}\left(A_{n}\right)\left(u_{n}\right)+\varepsilon C,
$$

где $C=\{r \in \mathbb{R} ;|r|<1\}$. Из этого включения вытекает, что $\left|r_{n}-r\right|<\varepsilon, n>N$. Следовательно, последовательность $r_{n}, n \geqslant 1$, сходится к $r$. Лемма доказана. 
Лемма 2.3. Пусть $F: T \rightarrow c b X-$ измеримое отображение и $и(t)-$ его измеримый селектор. Тогда функиии $d^{s}(F)(u(t)), g^{s}(F)(t)$ и многозначное отображение $L^{S}(F)(u(t))$, определенные по формулам (2.1)-(2.3), измеримы.

ДокАЗАтЕльство. Рассмотрим многозначное отображение $F_{0}(t)$, определенное по правилу

$$
F_{0}(t)=\{x \in X ; x \in F(t)-u(t),-x \in F(t)-u(t)\},
$$

которое является измеримым отображением [20] со значениями в $c b X$. Простая проверка показывает, что

$$
d^{s}(F)(u(t))=\sup \left\{\left\langle 2 z, x_{s}^{\prime}\right\rangle ; z \in F_{0}(t)\right\} .
$$

Теперь измеримость $d^{s}(F(u)(t))$ следует из (2.6) и теоремы 2.2 [22]. Измеримость функции $g^{s}(F)(t)$ также следует из этой теоремы, если учесть, что

$$
g^{s}(F)(t)=\sup \left\{\left\langle x-y, x_{s}^{\prime}\right\rangle ;(x, y) \in F(t) \times F(t)\right\}
$$

и отображение $F(t) \times F(t)$ измеримо [20]. Измеримость отображения $L^{s}(F)(u(t))$ непосредственно вытекает из измеримости функций $d^{s}(F)(u(t))$ и $g^{s}(F)(t)$. Лемма доказана.

Из леммы 2.3 и свойств измеримых отображений с компактными значениями следует, что если $F_{n}, F: T \rightarrow c b X, n \geqslant 1$, измеримы и $u_{n}(t), u(t), r(t)$ - измеримые селекторы отображений $F_{n}(t), F(t), L^{s}(F)(u(t))$, то существует последовательность измеримых селекторов $r_{n}(t), n \geqslant 1$, отображений $L^{s}\left(F_{n}\right)\left(u_{n}(t)\right)$ такая, что

$$
\left|r(t)-r_{n}(t)\right|=\min \left\{|r(t)-k| ; k \in L^{s}\left(F_{n}\right)\left(u_{n}(t)\right)\right\} .
$$

Лемма 2.4. Пусть при почти всех $t \in T$ последовательность $F_{n}: T \rightarrow$ $c b X, n \geqslant 1$, измеримых отображсений сходится к измеримому отображению $F: T \rightarrow c b X$ в метрике Хаусдорфа, а последовательность $u_{n}(t), n \geqslant 1$, измеримых селекторов $F_{n}(t)$ сходится $\kappa$ измеримому селектору и $(t)$ отображения $F(t)$. Тогда для любого измеримого селектора $r(t)$ отображения $L^{s}(F)(u(t))$ последовательность измеримых селекторов $r_{n}(t), n \geqslant 1$, отображений $L^{s}\left(F_{n}\right)\left(u_{n}(t)\right)$, удовлетворяющая $(2.7)$, почти всюду сходится $\kappa r(t)$.

Лемма непосредственно следует из леммы 2.2 .

3. Пусть $F: T \rightarrow b X$ - измеримое, интегрально ограниченное отображение. Обозначим через $S(F)$ совокупность всех измеримых селекторов отображения $F$. Тогда $S(F)$ является элементом пространства $d b L_{1}[21]$.

Лемма 3.1. Пусть $F_{1}, F_{2}: T \rightarrow b X-$ измеримые, интегрально ограниченнъе отображсения. Тогда

$$
\operatorname{dist}_{L_{1}}\left(S\left(F_{1}\right), S\left(F_{2}\right)\right) \leqslant \int_{T} \operatorname{dist}\left(F_{1}(t), F_{2}(t)\right) d \mu_{0} \leqslant 2 \operatorname{dist}_{L_{1}}\left(S\left(F_{1}, S\left(F_{2}\right)\right) .\right.
$$


ДокАЗАТЕЛЬСтво. Не оговаривая особо, отметим, что все функции, фигурирующие в доказательстве, будут интегрируемыми в силу леммы 2.1 [21].

Возьмем произвольный элемент $x(\cdot) \in S\left(F_{1}\right)$. Тогда, воспользовавшись теоремой $2.2[21]$, получим

$$
\begin{aligned}
\mathrm{d}_{L_{1}}\left(x(\cdot), S\left(F_{2}\right)\right) & =\inf \left\{\|x(\cdot)-y(\cdot)\|_{L_{1}} ; y \in S\left(F_{1}\right)\right\} \\
& =\int_{T} \mathrm{~d}\left(x(t), F_{2}(t)\right) d \mu_{0} \leqslant \int_{T} \operatorname{dist}\left(F_{1}(t), F_{2}(t)\right) d \mu_{0} .
\end{aligned}
$$

Аналогично, для любого элемента $y(\cdot) \in S\left(F_{2}\right)$ имеем

$$
\mathrm{d}_{L_{1}}\left(y(\cdot), S\left(F_{1}\right)\right) \leqslant \int_{T} \operatorname{dist}\left(F_{1}(t), F_{2}(t)\right) d \mu_{0} .
$$

Из неравенств (3.2), (3.3) сразу вытекает левое неравенство (3.1).

Перейдем к доказательству правого неравенства (3.1). Из определения функции $\operatorname{dist}\left(F_{1}(t), F_{2}(t)\right)$ следует, что существует множество $E \in \Sigma$ такое, что

$$
\begin{aligned}
\operatorname{dist}\left(F_{1}(t), F_{2}(t)\right) & =\left\{\operatorname{supd}\left(x, F_{1}(t)\right) ; x \in F_{2}(t)\right\}, & & t \in E, \\
\operatorname{dist}\left(F_{1}(t), F_{2}(t)\right) & =\left\{\operatorname{supd}\left(y, F_{2}(t)\right) ; y \in F_{1}(t)\right\}, & & t \in T \backslash E .
\end{aligned}
$$

Воспользовавшись теоремой 2.2 [21], получаем,

$$
\begin{aligned}
\int_{E} \sup \left\{\mathrm{d}\left(x, F_{1}(t)\right) ; x \in F_{2}(t)\right\} d \mu_{0} & =\sup _{x(\cdot) \in S\left(F_{2}\right)} \int_{E} \mathrm{~d}\left(x(t), F_{1}(t)\right) d \mu_{0} \\
& \leqslant \sup _{x(\cdot) \in S\left(F_{2}\right)} \int_{T} \mathrm{~d}\left(x(t), F_{1}(t)\right) d \mu_{0} \\
& =\sup _{x(\cdot) \in S\left(F_{2}\right)} \mathrm{d}_{L_{1}}\left(x(\cdot), S\left(F_{1}\right)\right) \\
& \leqslant \operatorname{dist}_{L_{1}}\left(S\left(F_{1}\right), S\left(F_{2}\right)\right) .
\end{aligned}
$$

Аналогично,

$$
\int_{T \backslash E} \sup \left\{\mathrm{d}\left(y, F_{2}(t)\right) ; y \in F_{1}(t)\right\} d \mu_{0} \leqslant \operatorname{dist}_{L_{1}}\left(S\left(F_{1}\right), S\left(F_{2}\right)\right) .
$$

Складывая неравенства (3.4), (3.5), приходим к правому неравенству (3.1). Лемма доказана.

Пусть $\Gamma \in d c b L_{1}$. Тогда в соответствии с теоремами $3.1,3.2$ [21] существует единственное с точностью до множества нулевой меры, измеримое, интегрально ограниченное отображение $F: T \rightarrow c b X$ такое, что

$$
\Gamma=S(F) .
$$

Для множества $\Gamma \in d c b L_{1}$ и элемента $u(\cdot) \in \Gamma$ положим

$$
\begin{aligned}
D^{s}(\Gamma)(u(\cdot)) & =\sup \left\{\left\langle y(\cdot)-z(\cdot), x_{s}^{\prime} \chi(\cdot)\right\rangle ; y(\cdot), z(\cdot) \in \Gamma, u(\cdot)=\frac{y(\cdot)+z(\cdot)}{2}\right\} \\
G^{s}(\Gamma) & =\sup \left\{\left\langle y(\cdot)-z(\cdot), x_{s}^{\prime} \chi(\cdot)\right\rangle ; y(\cdot), z(\cdot) \in \Gamma\right\}
\end{aligned}
$$


где $\chi(\cdot)$ - характеристическая функция множества $T$. Для многозначного отображения $F: T \rightarrow c b X$, связанного с множеством $\Gamma$ равенством (3.6), и его селектора $u(\cdot)$ определим по формулам $(2.1)-(2.3)$ функции $d^{s}(F)(u(t)), g^{s}(F)(t)$ и многозначное отображение $L^{s}(F)(u(t))$. Из интегральной ограниченности отображения $F(t)$ и леммы 2.3 следует, что функции $d^{s}(F)(u(t)), g^{s}(F)(t)$ суммируемы, а многозначное отображение $L^{s}(F)(u(t))$ измеримо и интегрально ограничено.

Поскольку множество $\left\{x_{s}^{\prime} \chi(\cdot)\right\}_{1}^{\infty} \subset L_{\infty}(T, X)$ не разделяет точки пространства $L_{1}$, то свойства функции $D^{s}(\Gamma)(u(\cdot))$ формально не следуют из леммы 2.1. Тем не менее справедлива

Лемма 3.2. Пусть $\Gamma \in d c b L_{1} u u(\cdot) \in \Gamma$. Тогда для любого $s \geqslant 1$

$$
\begin{aligned}
D^{s}(\Gamma)(u(\cdot)) & =\int_{T} d^{s}(F)(u(t)) d \mu_{0} \\
G^{s}(\Gamma) & =\int_{T} g^{s}(F)(t) d \mu_{0}
\end{aligned}
$$

3) $u(\cdot) \in \operatorname{ext} \Gamma$ тогда и только тогда, когда $D^{s}(\Gamma)(u(\cdot))=0$ для всех $s \geqslant 1$;

4) если последовательность $\Gamma_{n} \in d c b L_{1}, n \geqslant 1$, сходится в метрике Хаусдорфа $\operatorname{dist}_{L_{1}}(\cdot, \cdot)$, а последовательность $u_{n}(\cdot) \in \Gamma_{n}, n \geqslant 1$, сходится в $L_{1}$ $\kappa u(\cdot) \in \Gamma, m o$

$$
\limsup _{n \rightarrow \infty} D^{s}\left(\Gamma_{n}\right)\left(u_{n}(\cdot)\right) \leqslant D^{s}(\Gamma)(u(\cdot))
$$

ДокаЗАТЕЛЬство. Рассмотрим множество

$$
\Gamma_{0}=\left\{y(\cdot) \in L_{1} ; y(\cdot) \in \Gamma-u(\cdot),-y(\cdot) \in \Gamma-u(\cdot)\right\}
$$

и многозначное отображение $F_{0}(t)$, определенное по правилу $(2.5)$. Тогда $\Gamma_{0} \in$ $d c b L_{1}$ и $\Gamma_{0}=S\left(F_{0}\right)$. Непосредственной проверкой убеждаемся, что

$$
D^{s}(\Gamma)(u(\cdot))=\sup \left\{\left\langle 2 y(\cdot), x_{s}^{\prime} \chi(\cdot)\right\rangle ; y(\cdot) \in \Gamma_{0}\right\}
$$

и справедливо равенство (2.6). Поскольку для любого $y(\cdot) \in \Gamma_{0}$

$$
\left\langle 2 y(\cdot), x_{s}^{\prime} \chi(\cdot)\right\rangle=\int_{T}\left\langle 2 y(t), x_{s}^{\prime}\right\rangle d \mu_{0}
$$

то равенство (3.7) следует из (3.9), (2.6), (3.10) и теоремы 2.2 [21].

Равенство (3.8) доказывается аналогичным образом с использованием теоремы $2.2[21]$.

Перейдем к доказательству утверждения 3$)$. Если $u(\cdot) \in \operatorname{ext} \Gamma$, то равенства $D^{s}(\Gamma)(u(\cdot))=0, s \geqslant 1$, следуют из определения экстремальной точки.

Пусть теперь $D^{s}(\Gamma)(u(\cdot))=0, s \geqslant 1$. Предположим, что $u(\cdot)$ не является экстремальной точкой. Тогда найдутся элементы $y(\cdot), z(\cdot) \in \Gamma, y(\cdot) \neq z(\cdot)$, такие, что $u(\cdot)=(y(\cdot)+z(\cdot)) / 2$. Для $\varepsilon>0$ выберем, используя равенство $(3.7)$, 
компактное подмножество $T_{\varepsilon} \subset T, \mu\left(T \backslash T_{\varepsilon}\right) \leqslant \varepsilon$, такое, что $u(t), y(t), z(t)$ непрерывны на $T_{\varepsilon}, y(t) \neq z(t), u(t)=(y(t)+z(t)) / 2, u(t), y(t), z(t) \in F(t), t \in T_{\varepsilon}$, и $d^{s}(F)(u(t))=0, t \in T_{\varepsilon}, s \geqslant 1$. Так как множество $\left\{x_{s}^{\prime}\right\}_{1}^{\infty}$ разделяет точки пространства $X$, то для любой точки $t \in T_{\varepsilon}$ найдется номер $s \geqslant 1$, зависящий от $t$, который мы будем обозначать $s(t)$, такой, что $\left\langle y(t)-z(t), x_{s(t)}^{\prime}\right\rangle>0$. Тогда в силу непрерывности $y(t), z(t)$ на $T_{\varepsilon}$, найдется окрестность $V(t)$ в $T_{\varepsilon}$ точки $t$ такая, что $\left\langle y(\tau)-z(\tau), x_{s(t)}^{\prime}\right\rangle>0$ для всех $\tau \in V(t)$. Поскольку множества $V(t)$, $t \in T_{\varepsilon}$, образуют открытое покрытие компакта $T_{\varepsilon}$, то существует $t^{*} \in T_{\varepsilon}$ такое, что $\mu_{0}\left(V\left(t^{*}\right)\right)>0$.

Из определения меры Радона следует, что сушествует компакт $T_{0} \subset V\left(t^{*}\right)$ такой, что $\mu_{0}\left(T_{0}\right)>0$. Тогда для $s^{*}=s\left(t^{*}\right)$

$$
0<\int_{T_{0}}\left\langle y(\tau)-z(\tau), x_{s^{*}}^{\prime}\right\rangle d \mu_{0} \leqslant \int_{T} d^{s^{*}}(F)(u(\tau)) d \mu_{0}=D^{s^{*}}(\Gamma)(u(\cdot))=0,
$$

что противоречит нашему предположению. Тем самым утверждение 3 ) доказано.

Так как $L_{1}$ сепарабельно, то утверждение 4 ) следует из леммы 2.1. Лемма доказана.

СлЕДСТвИЕ 3.1. Пусть $\Gamma \in d c b L_{1}$. Точка $u(\cdot) \in \operatorname{ext} \Gamma$ тогда и только тогда, когда $u(t) \in \operatorname{ext} F(t)$ при почти всех $t \in T$.

Следствие вытекает из утверждения б) леммы 2.1 и утверждений 1$), 3)$ леммы 3.2.

Пусть $M$ - сепарабельное метрическое пространство и $\Gamma: M \rightarrow d c b L_{1}-$ непрерывное отображение, $u: M \rightarrow L_{1}$ - его непрерывный селектор, который всегда существует [3]. Обозначим через $F(\xi), \xi \in M$, многозначное отображение $F(\xi): T \rightarrow c b X$, связанное с $\Gamma(\xi)$ равенством (3.6). Тогда на $M$ будут определены функции

$$
\begin{gathered}
D^{s}(u(\xi))=D^{s}(\Gamma(\xi))(u(\xi)), \quad G^{s}(\xi)=G^{s}(\Gamma(\xi)), \quad D^{s}(u(\xi)) \leqslant G^{s}(\xi), \\
d^{s}(u(\xi))(t)=d^{s}(F(\xi))(u(\xi)(t)), \quad g^{s}(\xi)(t)=g^{s}(F(\xi))(t), \quad \xi \in M,
\end{gathered}
$$

и многозначное отображение

$$
L^{s}(u(\xi))(t)=L^{s}(F(\xi))(u(\xi))(t), \quad \xi \in M .
$$

Согласно лемме 3.1

$$
\begin{aligned}
D^{s}(u(\xi)) & =\int_{T} d^{s}(u(\xi))(t) d \mu_{0}, \\
G^{s}(\xi) & =\int_{T} g^{s}(\xi)(t) d \mu_{0}, \quad \xi \in M .
\end{aligned}
$$

Для каждого $\xi \in M$ положим $\mathscr{L}^{s}(u(\xi))=S\left(L^{s}(u(\xi))\right)$.

Теорема 3.1. Пусть $\Gamma: M \rightarrow d c b L_{1}$ непрерьвно. Тогда отображсение $\xi \rightarrow$ $\mathscr{L}^{s}(u(\xi))$ является полунепрерывным снизу многозначныц отображением из $M$ в $d \operatorname{conv}\left(L_{1}(T, R)(\sigma)\right)$. 
ДокАЗАТЕЛЬство. Для фиксированного $\xi \in M$ и каждого измеримого селектора $r(t)$ отображения $L^{s}(u(\xi))$ почти всюду справедливо неравенство

$$
0 \leqslant d^{s}(u(\xi))(t) \leqslant r(t) \leqslant g^{s}(\xi)(t) \leqslant 2\|F(\xi)(t)\|
$$

Из этого неравенства следует, что $\mathscr{L}^{s}(u(\xi)) \in d \operatorname{conv}\left(L_{1}(T, R)(\sigma)\right)$.

Пусть $\xi_{0} \in M$ - фиксированная точка. Для доказательства полунепрерьвности снизу отображения $\mathscr{L}^{s}(u(\xi))$ в точке $\xi_{0}$ достаточно показать, что для любой последовательности $\xi_{n} \rightarrow \xi_{0}, n \geqslant 1$, и любого элемента $r\left(\xi_{0}\right) \in \mathscr{L}^{s}\left(u\left(\xi_{0}\right)\right)$ сушествует последовательность элементов $r\left(\xi_{n}\right) \in \mathscr{L}^{s}\left(u\left(\xi_{n}\right)\right), n \geqslant 1$, сходящаяся к $r\left(\xi_{0}\right)$ в топологии пространства $L_{1}(T, R)$. Так как $r\left(\xi_{0}\right) \in \mathscr{L}^{s}\left(u\left(\xi_{0}\right)\right)$, то $r\left(\xi_{0}\right)(t) \in L^{s}\left(u\left(\xi_{0}\right)\right)(t), t \in T$. Тогда сушествует последовательность измеримых селекторов $r\left(\xi_{n}\right)(t) \in L^{s}\left(u\left(\xi_{n}\right)\right)(t), n \geqslant 1$, такая, что

$$
\left|r\left(\xi_{0}\right)(t)-r\left(\xi_{n}\right)(t)\right|=\min \left\{\left|r\left(\xi_{0}\right)(t)-k\right| ; k \in L^{s}\left(u\left(\xi_{n}\right)\right)(t)\right\}, \quad t \in T .
$$

Так как последовательность $\Gamma\left(\xi_{n}\right), n \geqslant 1$, сходится к $\Gamma\left(\xi_{0}\right)$ в метрике $\operatorname{dist}_{L_{1}}(\cdot, \cdot)$, а последовательность $u\left(\xi_{n}\right), n \geqslant 1$, сходится к $u\left(\xi_{0}\right)$ в $L_{1}$, то в соответствии с леммой 3.1 сушествует подпоследовательность $\xi_{n_{k}}, k \geqslant 1$, последовательности $\xi_{n}$, $n \geqslant 1$, такая, что $F\left(\xi_{n_{k}}\right)(t)$ сходится к $F\left(\xi_{0}\right)(t)$ в метрике $\operatorname{dist}(\cdot, \cdot)$, а $u\left(\xi_{n_{k}}\right)(t)$, $k \geqslant 1$, сходится к $u\left(\xi_{0}\right)(t)$ при почти всех $t \in T$. Тогда в соответствии с леммой 2.4 последовательность $r\left(\xi_{n_{k}}\right)(t), k \geqslant 1$, при почти всех $t \in T$ сходится к $r\left(\xi_{0}\right)(t)$.

Из неравенства

$$
\int_{E}\left\|F\left(\xi_{n}\right)(t)\right\| d \mu_{0} \leqslant \int_{E} \operatorname{dist}\left(F\left(\xi_{n}\right)(t), F\left(\xi_{0}\right)(t)\right) d \mu_{0}+\int_{E}\left\|F\left(\xi_{0}\right)(t)\right\| d \mu_{0}, \quad E \in \Sigma,
$$

и леммы 3.1 следует, что последовательность $\left\|F\left(\xi_{n}\right)(t)\right\|, n \geqslant 1$, равномерно интегрируема. Тогда в соответствии с (3.13) последовательность $r\left(\xi_{n_{k}}\right)(t), k \geqslant 1$, также равномерно интегрируема. Следовательно, согласно утверждению 1.3 последовательность $r\left(\xi_{n_{k}}\right), k \geqslant 1$, сходится к $r\left(\xi_{0}\right)$ в $L_{1}(T, R)$.

Предположим, что сама последовательность $r\left(\xi_{n}\right), n \geqslant 1$, не сходится к $r\left(\xi_{0}\right)$ в $L_{1}(T, R)$. Тогда сушествует подпоследовательность $r\left(\xi_{n_{k}}\right), k \geqslant 1$, последовательности $r\left(\xi_{n}\right), n \geqslant 1$, такая, что любая подпоследовательность последовательности $r\left(\xi_{n_{k}}\right), k \geqslant 1$, не сходится к $r\left(\xi_{0}\right)$. Применяя к последовательности $r\left(\xi_{n_{k}}\right)$, $k \geqslant 1$, рассуждения, приведенные выше, приходим к противоречию. Теорема доказана.

Обозначим через $\Lambda^{s}(u(\cdot))$ совокупность всех непрерывных селекторов отображения $\mathscr{L}^{s}(u(\xi))$. Поскольку отображение $\mathscr{L}^{s}(u(\xi))$ полунепрерывно снизу и принимает значения в $d \operatorname{conv}\left(L_{1}(T, R)(\sigma)\right)$, то множество $\Lambda^{s}(u(\cdot)) \neq \varnothing$ и для любого элемента $r_{0} \in \mathscr{L}^{s}\left(u\left(\xi_{0}\right)\right)$ сушествует непрерывньй селектор $r(u(\xi))$ отображения $\mathscr{L}^{s}(u(\xi)), \xi \in M$, такой, что $r\left(u\left(\xi_{0}\right)\right)=r_{0}$ [3]. Отсюда и из $(3.11),(3.12)$ вытекает 
ЛЕмма 3.3. Пусть отображсение $\Gamma: M \rightarrow d c b L_{1}$ удовлетворяет всем предполохсениям теоремы 3.1. Тогда

1) $\mathscr{L}^{s}(u(\xi))=\left\{r(u(\xi)) ; r(u(\cdot)) \in \Lambda^{s}(u(\cdot))\right\}, \quad \xi \in M$;

2) $D^{s}(u(\xi)) \leqslant \int_{T} r(u(\xi))(t) d \mu_{0} \leqslant G^{s}(\xi), \quad \xi \in M$;

3) для любого $\xi_{0} \in M$ существует әлемент $r(u(\cdot)) \in \Lambda^{s}(u(\cdot))$ такой, что

$$
D^{s}\left(u\left(\xi_{0}\right)\right)=\int_{T} r\left(u\left(\xi_{0}\right)\right)(t) d \mu_{0} .
$$

4. Для $A \in c b X$ и $x^{\prime} \in X^{\prime}, x^{\prime} \neq 0$, положим $M\left(A, x^{\prime}\right)=\sup \left\{\left\langle x, x^{\prime}\right\rangle ; x \in A\right\}$. Если $\alpha>0$, то

$$
S\left(A, x^{\prime}, \alpha\right)=\left\{x \in A ;\left\langle x, x^{\prime}\right\rangle>M\left(A, x^{\prime}\right)-\alpha\right\} .
$$

Точка $x \in A$ называется строго выступающей (strongly exposed), если сушествует $x^{\prime} \in X^{\prime}$ такой, что $\left\langle x, x^{\prime}\right\rangle \geqslant\left\langle y, x^{\prime}\right\rangle$ для всех $y \in A, y \neq x$, и множества $\left\{S\left(A, x^{\prime}, \alpha\right) ; \alpha>0\right\}$ являются базой окрестностей в $A$ точки $x$ в топологии, индуцированной на $A$ из $X[23]$.

Совокупность всех строго выступающих точек множества $A$ обозначим st $A$.

Скажем, что множество $A \in c b X$ обладает свойством Радона-Никодима $(\mathrm{R}-\mathrm{N}$ свойство), если каждое замкнутое выпуклое подмножество $C \subset A$ представимо в виде $C=\overline{\text { co st } C}$ [23].

Хорошо известно, что любой элемент $A \in \operatorname{conv} X(\sigma)$ обладает $\mathrm{R}-\mathrm{N}$ свойством [23].

ПРЕДПОЛОЖЕНИЕ (Н1). Для непрерьвного отображения $\Gamma: M \rightarrow d c b L_{1}$ множество $\operatorname{ext} \Gamma(\xi) \neq \varnothing, \xi \in M$, и существует полунепрерывное снизу многозначное отображение $\mathscr{F}: M \rightarrow L_{1}$ такое, что $\mathscr{F}(\xi) \subset \operatorname{ext} \Gamma(\xi), \xi \in M$.

ПреДПОЛОЖЕНИЕ (Н2). Для непрербъного отображения $Г: M \rightarrow d c b L_{1}$ множество $\operatorname{ext} \Gamma(\xi) \neq \varnothing, \xi \in M$, и существует полунепрерьвное снизу многозначное отображсение $\mathscr{F}: M \rightarrow L_{1}$ такое, что $\mathscr{F}(\xi) \subset \overline{\operatorname{ext}} \Gamma(\xi), \xi \in M$, где черта означает замыкание множества $\operatorname{ext} \Gamma(\xi)$.

Приведем пример отображения Г, удовлетворяюшего предположению (Н1).

УТВЕРЖДЕНИЕ 4.1. Пусть $\Gamma: M \rightarrow d c b L_{1}$ непрерьвно и значениями $\Gamma$ являются множества, обладающие свойством R-N. Тогда Г удовлетворяет предположению (H1).

Так как $\Gamma(\xi), \xi \in M$, обладает свойством $\mathrm{R}-\mathrm{N}$, то st $\subset \operatorname{ext} \Gamma(\xi) \neq \varnothing$ [23]. Coгласно лемме $2[16]$ для непрерьвного отображения $\Gamma: M \rightarrow d c b L_{1}$ отображение st $\Gamma(\xi), \xi \in M$, полунепрерывно снизу. Теперь в качестве отображения $\mathscr{F}$, фигурируюшего в предположении (H1), можно взять отображение st $\Gamma$.

Пусть непрерывное отображение $\Gamma: M \rightarrow d c b L_{1}$ удовлетворяет предположению (H1) и $\mathscr{F}: M \rightarrow L_{1}, \mathscr{F}(\xi) \subset \operatorname{ext} \Gamma(\xi), \xi \in M,-$ полунепрерывное снизу отображение. Обозначим через $\operatorname{dec} \mathscr{F}(\xi)$ разложимую оболочку множества $\mathscr{F}(\xi)$, т.е. 
наименьшее по включению разложимое множество, содержащее $\mathscr{F}(\xi)$. Из следствия 3.1 вытекает, что множество ext $\Gamma(\xi), \xi \in M$, разложимо. Поэтому $\operatorname{dec} \mathscr{F}(\xi) \subset$ $\operatorname{ext} \Gamma(\xi), \xi \in M$. Согласно лемме $3.2[4]$ множества $\overline{\operatorname{dec}} \mathscr{F}(\xi), \overline{\operatorname{ext}} \Gamma(\xi), \xi \in M$, где черта вверху означает замькание в $L_{1}$, разложимы и отображение $\overline{\operatorname{dec}} \mathscr{F}(\xi)$ полунепрерывно снизу. Более того, отображение $\overline{\mathrm{dec}} \mathscr{F}$ имеет непрерывные селекторы, и для любых $\xi_{0} \in M, w_{0} \in \overline{\operatorname{dec}} \mathscr{F}\left(\xi_{0}\right)$ существует непрерьвный селектор $w(\cdot)$ отображения $\overline{\operatorname{dec}} \mathscr{F}$ такой, что $w\left(\xi_{0}\right)=w_{0}[3]$.

ТеОРема 4.1. Пусть $\Gamma: M \rightarrow d c b L_{1}$ непрерьвно и удовлетворяет предположению $(\mathrm{H} 1), w(\cdot)$ - непрерывный селектор отображсения $\overline{\operatorname{dec}} \mathscr{F} u \varphi_{i}: M \rightarrow$ $(0,+\infty), i=1,2,-$ полунепрерывные снизу функиии. Тогда для любого $s \geqslant 1$ существует непрерывный селектор $v(\cdot)$ отображсения $\overline{\operatorname{dec}} \mathscr{F}$ такой, что

$$
\begin{gathered}
\|w(\xi)-v(\xi)\|_{L_{1}}<\varphi_{1}(\xi), \\
D^{s}(v(\xi))<\varphi_{2}(\xi), \quad \xi \in M
\end{gathered}
$$

ДокАЗАТЕльство. Обозначим через $\mathscr{G}$ совокупность всех непрерывных селекторов отображения $\overline{\operatorname{dec}} \mathscr{F}(\xi)$. Для каждого элемента $v(\cdot) \in \mathscr{G}$ возьмем совокупность $\Lambda^{s}(v(\cdot))$ всех непрерьвных селекторов отображения $\mathscr{L}^{s}(v(\cdot))$ (см. леммy 3.3).

Пусть $B$ - единичный открытый шар в $L_{1}$ с центром в нуле и

$$
c(\xi)=\min \left\{\varphi_{1}(\xi), \varphi_{2}(\xi)\right\}, \quad \xi \in M .
$$

Выберем непрерывную функцию $\nu: M \rightarrow \mathbb{R}^{+}$такую, что $0<\nu(\xi)<c(\xi)$. Такая функция сушествует согласно лемме 3.6 [24], поскольку функция $c(\xi)$ полунепрерывна снизу. Для каждой пары $v(\cdot) \in \mathscr{G}, r(v(\cdot)) \in \Lambda^{s}(v(\cdot))$ положим

$\mathscr{U}_{v, r(v)}=\left\{\xi \in M ; v(\xi) \in w(\xi)+\left(\varphi_{1}(\xi)-\nu(\xi)\right) B, \int_{T} r(v(\xi))(t) d \mu_{0}<\varphi_{2}(\xi)-\nu(\xi)\right\}$.

Так как функции $\varphi_{1}(\xi)-\nu(\xi), \varphi_{2}(\xi)-\nu(\xi)$ полунепрерывны снизу, функции $v(\xi)$, $w(\xi)$ непрерывны в $L_{1}(T, X)$, a $r(v(\xi))$ непрерывна в $L_{1}(T, R)$, то множества $\mathscr{U}_{v}, r(v)$ открыты в $M$.

Покажем, что

$$
M=\left\{\bigcup \mathscr{U}_{v, r(v)} ; v(\cdot) \in \mathscr{G}, r(v(\cdot)) \in \Lambda^{s}(v(\cdot))\right\} .
$$

Пусть $\xi_{0} \in M$. Тогда в любой окрестности элемента $w\left(\xi_{0}\right)$ найдется элемент $v_{0} \in$ $\operatorname{dec} \mathscr{F}\left(\xi_{0}\right)$, через который проходит непрерывный селектор $v(\xi)$ отображения $\overline{\operatorname{dec}} \mathscr{F}$ такой, что $v\left(\xi_{0}\right)=v_{0}$. Поскольку $v_{0} \in \operatorname{ext} \Gamma\left(\xi_{0}\right)$, то в соответствии с леммой 3.2 $D^{s}\left(v\left(\xi_{0}\right)\right)=0$. Согласно лемме 3.3 для $\xi_{0}$ сушествует элемент $r(v(\cdot)) \in \Lambda^{s}(v(\cdot))$ такой, что

$$
D^{s}\left(v\left(\xi_{0}\right)\right)=\int_{T} r\left(v\left(\xi_{0}\right)\right)(t) d \mu_{0}=0
$$

Поэтому $\xi_{0} \in \mathscr{U}_{v, r(v)}$ при указанных выше $v(\cdot) \in \mathscr{G}, r(v(\cdot)) \in \Lambda^{s}(v(\cdot))$. 
Пусть $\left\{V_{n}\right\}_{1}^{\infty}$ - счетное локально конечное измельчение покрытия $\mathscr{U}_{v, r(v)}$ и $\left\{e_{n}\right\}_{1}^{\infty}$ - непрерывное локально конечное разбиение единицы, подчиненное $\left\{V_{n}\right\}_{1}^{\infty}$, такое, что $\operatorname{supp} e_{n} \subset V_{n}$, где $\operatorname{supp} e_{n}-$ носитель функции $V_{n}$ [26]. Тогда для каждого $n \geqslant 1$ найдутся $v_{n}(\cdot) \in \mathscr{G}, r_{n}\left(v_{n}(\cdot)\right) \in \Lambda^{s}\left(v_{n}(\cdot)\right)$, при которых $V_{n} \subset \mathscr{U}_{v_{n}, r\left(v_{n}\right)}[26]$. Так как $\operatorname{supp} e_{n}$ является замкнутьм подмножеством пространства $M$, то сушествует непрерывная функция $h\left[\operatorname{supp} e_{n}, V_{n}\right]$, удовлетворяющая $0 \leqslant h\left[\operatorname{supp} e_{n}, V_{n}\right](\xi) \leqslant 1$, равная 1 на supp $e_{n}$ и нулю вне $V_{n}$. В силу утверждения 1.2 сушествует функция $f: T \times X \times M \rightarrow X^{\prime}$, слабо измеримая по $t$, непрерывная по $(x, \xi)$ в топологии пространства $X^{\prime}(\sigma)$ и такая, что

$$
\|x\| \leqslant\langle x, f(t, x, \xi)\rangle+\frac{\nu(\xi)}{2 k(\xi) \mu_{0}(T)}, \quad f(t, x, \xi) \in \partial(\|x\|),
$$

почти всюду для любых $x \in X, \xi \in M$, где $k(\xi)=\sum_{n=1}^{\infty} h\left[\operatorname{supp} e_{n}, V_{n}\right](\xi)$.

Поскольку supp $e_{n} \subset V_{n}, n \geqslant 1$, и $\left\{V_{n}\right\}_{1}^{\infty}$ - локально конечное покрытие $M$, то функция $k(\xi)>0, \xi \in M$, определена и непрерывна. Из свойств $f(t, x, \xi)$ следует, что для любого $x(\cdot) \in L_{1}$ функция $\langle x(t), f(t, x(t), \xi)\rangle$ интегрируема.

Для каждых $x(\cdot) \in L_{1}, \xi \in M$, определим числовую меру $\Re_{1}(x(\cdot), \xi)$,

$$
\Re_{1}(x(\cdot), \xi)(E)=\int_{E}\langle x(t), f(t, x(t), \xi)\rangle d \mu_{0}, \quad E \in \Sigma .
$$

Тогда отображение $\Re_{1}: L_{1} \times M \rightarrow \mathscr{M}$ непрерывно. Доказательство этого утверждения можно найти в $[6$, c. 546]. Аналогично, для каждой пары $(v(\cdot), r(v(\cdot))) \in$ $\mathscr{G} \times \Lambda^{s}(v(\cdot))$ и $\xi \in M$ определим меру

$$
\left.\Re_{2}(v(\xi), r(v(\xi)))(E)=\int_{E} r(v(\xi))(t)\right\rangle d \mu_{0}, \quad E \in \Sigma .
$$

Тогда отображение $\Re_{2}(v(\cdot), r(v(\cdot))): M \rightarrow \mathscr{M}$ непрерьвно, что следует из определения полной вариации меры на $T$.

Для каждого $n \geqslant 1, \xi \in M$ положим

$$
\mathscr{P}_{n}(\xi)=\frac{k(\xi)}{\nu(\xi)} \cdot\left\{\Re_{1}\left(v_{n}(\xi)-w(\xi), \xi\right) \cup \Re_{2}\left(v_{n}(\xi), r_{n}\left(v_{n}(\xi)\right)\right)(E)\right\} .
$$

В силу непрерывности по $\xi$ всех функций, входящих в выражение для $\mathscr{P}_{n}(\xi)$, многозначное отображение $\mathscr{P}_{n}: M \rightarrow \mathscr{M}$ непрерывно и его значениями являются компактные множества, состояшие из двух элементов. Воспользовавшись утверждением 1.1, получаем последовательность непрерывных отображений $\mathscr{B}_{n}(\xi), n \geqslant 1$, со свойствами:

1) $\left\{\mathscr{B}_{n}(\xi)\right\}_{1}^{\infty}$ для каждого $\xi \in M$ является разбиением $T$, т.е. множества $\mathscr{B}_{n}(\xi)$ попарно не пересекаются и

$$
\mu_{0}\left(\bigcup_{n=1}^{\infty} \mathscr{B}_{n}(\xi)\right)=\mu_{0}(T) ;
$$

2 ) для каждого $n \geqslant 1$ и $\xi \in M$ множество $\mathscr{B}_{n}(\xi)=\varnothing$ тогда и только тогда, когда $e_{n}(\xi)=0$; 
3) для любых $\xi \in M, n \geqslant 1, \mu(\cdot) \in \mathscr{P}_{n}(\xi)$ справедливо неравенство

$$
\left|\mu\left(\mathscr{B}_{n}(\xi)\right)-e_{n}(\xi) \mu(T)\right|<\frac{1}{2} .
$$

Положим $v(\xi)=\sum_{n=1}^{\infty} v_{n}(\xi) \chi\left(\mathscr{B}_{n}(\xi)\right)$, где $\chi\left(\mathscr{B}_{n}(\xi)\right)$ - характеристическая функция множества $\mathscr{B}_{n}(\xi)$. Так как каждая из функций $v_{n}(\xi) \chi\left(\mathscr{B}_{n}(\xi)\right)$ непрерывна [2, утверждение 1.3], а $\left\{e_{n}\right\}_{1}^{\infty}$ - непрерывное локально конечное разбиение единицы, то из свойства 2$)$ отображений $\mathscr{B}_{n}(\xi)$ следует, что функция $v(\xi)$ определена и непрерывна. Более того, в силу разложимости множества $\overline{\operatorname{dec}} \mathscr{F}(\xi), \xi \in M$, следует, что $v(\xi) \in \overline{\operatorname{dec}} \mathscr{F}(\xi), \xi \in M$.

Покажем, что

$$
\|w(\xi)-v(\xi)\|_{L_{1}}<\varphi_{1}(\xi), \quad \xi \in M
$$

Для любых $n \geqslant 1, \xi \in M$ мера

$$
\mu(\cdot)=\frac{k(\xi)}{\nu(\xi)} \cdot \Re_{1}\left(v_{n}(\xi)-w(\xi), \xi\right)
$$

имеет вид

$$
\mu(E)=\frac{k(\xi)}{\nu(\xi)} \int_{E}\left\langle v_{n}(\xi)(t)-w(\xi)(t), f\left(t, v_{n}(\xi)(t)-w(\xi)(t), \xi\right)\right\rangle d \mu_{0}, \quad E \in \Sigma .
$$

Для нее неравенство (4.4) перепишется в виде

$$
\begin{aligned}
& \mid \int_{\mathscr{B}_{n}(\xi)}\left\langle v_{n}(\xi)(t)-w(\xi)(t), f\left(t, v_{n}(\xi)(t)-w(\xi)(t), \xi\right)\right\rangle d \mu_{0} \\
& \quad-e_{n}(\xi) \int_{T}\left\langle v_{n}(\xi)(t)-w(\xi)(t), f\left(t, v_{n}(\xi)(t)-w(\xi)(t), \xi\right)\right\rangle d \mu_{0} \mid<\frac{\nu(\xi)}{2 k(\xi)} .
\end{aligned}
$$

Тогда из этого неравенства и (4.3) получим

$$
\begin{aligned}
& \int_{\mathscr{B}_{n}(\xi)}\left\|v_{n}(\xi)(t)-w(\xi)(t)\right\| d \mu_{0} \\
& \quad \leqslant \int_{\mathscr{B}_{n}(\xi)}\left\langle v_{n}(\xi)(t)-w(\xi)(t), f\left(t, v_{n}(\xi)(t)-w(\xi)(t), \xi\right)\right\rangle d \mu_{0}+\frac{\nu(\xi)}{2 k(\xi)} \\
& \quad<e_{n}(\xi) \int_{T}\left\langle v_{n}(\xi)(t)-w(\xi)(t), f\left(t, v_{n}(\xi)(t)-w(\xi)(t), \xi\right)\right\rangle d \mu_{0}+\frac{\nu(\xi)}{k(\xi)} \\
& \quad<e_{n}(\xi)\left\|v_{n}(\xi)-w(\xi)\right\|_{L_{1}}+\frac{\nu(\xi)}{k(\xi)} .
\end{aligned}
$$

Если $e_{n}(\xi)>0$, то $\xi \in V_{n} \subset \mathscr{U}_{v_{n}, r_{n}\left(v_{n}\right)}$. Поэтому $\left\|v_{n}(\xi)-w(\xi)\right\|_{L_{1}}<\varphi_{1}(\xi)-\nu(\xi)$

и

$$
\int_{\mathscr{B}_{n}(\xi)}\left\|v_{n}(\xi)(t)-w(\xi)(t)\right\| d \mu_{0}<e_{n}(\xi)\left(\varphi_{1}(\xi)-\nu(\xi)\right)+\frac{\nu(\xi)}{k(\xi)} .
$$

Если $e_{n}(\xi)=0$, то $\mathscr{B}_{n}(\xi)=\varnothing$ и, следовательно, неравенство (4.5) выполняется автоматически. 
Для каждого $\xi \in M$ обозначим через $m(\xi)$ число номеров $n$ таких, что $\mathscr{B}_{n}(\xi) \neq \varnothing$. Тогда $e_{n}(\xi)>0$ и, поскольку $\left\{V_{n}\right\}_{1}^{\infty}$ - локально конечное покрытие, то $m(\xi)$ конечное число и $m(\xi) \leqslant k(\xi), \xi \in M$. Теперь из (4.5) вытекает

$$
\begin{aligned}
\left\|v_{n}(\xi)-w(\xi)\right\|_{L_{1}} & \leqslant \sum_{\left\{n, \mathscr{B}_{n}(\xi) \neq \varnothing\right\}} e_{n}(\xi)\left(\varphi_{1}(\xi)-\nu(\xi)\right)+\frac{m(\xi) \nu(\xi)}{k(\xi)} \\
& <\varphi_{1}(\xi)-\nu(\xi)+\nu(\xi)=\varphi_{1}(\xi), \quad \xi \in M .
\end{aligned}
$$

Тем самым неравенство (4.1) доказано.

Для любых $n \geqslant 1, \xi \in M$ мера

$$
\mu(\cdot)=\frac{k(\xi)}{\nu(\xi)} \cdot \Re_{2}\left(v_{n}(\xi), r_{n}\left(v_{n}(\xi)\right)\right)
$$

имеет вид

$$
\mu(E)=\frac{k(\xi)}{\nu(\xi)} \int_{E} r_{n}\left(v_{n}(\xi)\right)(t) d \mu_{0} .
$$

Для нее неравенство (4.4) перепишется в виде

$$
\int_{\mathscr{B}_{n}(\xi)} r_{n}\left(v_{n}(\xi)\right)(t) d \mu_{0}<e_{n}(\xi) \int_{T} r_{n}\left(v_{n}(\xi)\right)(t) d \mu_{0}+\frac{\nu(\xi)}{2 k(\xi)} .
$$

Если $e_{n}(\xi)>0$, то $\xi \in V_{n} \subset \mathscr{U}_{v_{n}, r_{n}\left(v_{n}\right)}$. Поэтому

$$
\int_{T} r_{n}\left(v_{n}(\xi)\right)(t) d \mu_{0}<\varphi_{2}(\xi)-\nu(\xi)
$$

Следовательно,

$$
\int_{\mathscr{B}_{n}(\xi)} r_{n}\left(v_{n}(\xi)\right)(t) d \mu_{0}<e_{n}(\xi)\left(\varphi_{2}(\xi)-\nu(\xi)\right)+\frac{\nu(\xi)}{2 k(\xi)} .
$$

Если $e_{n}(\xi)=0$, то $\mathscr{B}_{n}(\xi)=\varnothing$ и неравенство (4.6) выполняется автоматически.

Так как $d^{s}\left(v_{n}(\xi)\right)(t) \leqslant r_{n}\left(v_{n}(\xi)\right)(t)$ почти всюду на $T$, то

$$
\int_{\mathscr{B}_{n}(\xi)} d^{s}\left(v_{n}(\xi)\right)(t) d \mu_{0}<e_{n}(\xi)\left(\varphi_{2}(\xi)-\nu(\xi)\right)+\frac{\nu(\xi)}{2 k(\xi)} .
$$

Теперь из (3.11) и (4.7) получаем

$$
\begin{aligned}
D^{s}(v(\xi)) & =\sum_{\left\{n, \mathscr{B}_{n}(\xi) \neq \varnothing\right\}} \int_{\mathscr{B}_{n}(\xi)} d^{s}\left(v_{n}(\xi)\right)(t) d \mu_{0} \\
& <\varphi_{2}(\xi)-\nu(\xi)+\frac{\nu(\xi)}{2}<\varphi_{2}(\xi), \quad \xi \in M .
\end{aligned}
$$

Теорема доказана. 
Теорема 4.2. Пусть $\Gamma: M \rightarrow d c b L_{1}$ непрерывно и удовлетворяет предположению $(\mathrm{H} 2), w(\cdot)$ - непрерывный селектор отображения $\overline{\operatorname{dec}} \mathscr{F}$ и $\varphi_{i}: M \rightarrow$ $(0,+\infty), i=1,2,-$ полунепрерывные снизу функиии. Тогда для любого $s \geqslant 1$ существует непрерывный селектор $v(\cdot)$ отображсения $Г$ такой, что выполняются неравенства (4.1) и (4.2).

Доказательство дословно повторяет доказательство теоремы 4.1, следует только в качестве $\mathscr{G}$ взять совокупность всех непрерывных селекторов отображения $\Gamma$ и учесть, что отображение Г имеет разложимые значения.

5. Всюду в дальнейшем предполагается, что отображение $\Gamma: M \rightarrow d c b L_{1}$ ограничено на $M$.

Обозначим через $C\left(M, L_{1}\right)$ пространство всех непрерывных ограниченных функций из $M$ в $L_{1}$ с топологией равномерной сходимости на $M$, которое является полньмм метрическим пространством.

Пусть $C(M, \overline{\operatorname{dec}} \mathscr{F})$ и $C(M, \Gamma)$ - совокупности всех непрерывных селекторов отображений $\overline{\operatorname{dec}} \mathscr{F}$ и $\Gamma$ с топологией, индуцированной из $C\left(M, L_{1}\right)$. Тогда $C(M, \overline{\operatorname{dec}} \mathscr{F})$ и $C(M, \Gamma)$ являются замкнутыми подмножествами $C\left(M, L_{1}\right)$ и, следовательно, полными метрическими пространствами.

Возьмем фиксированное $\sigma$-компактное подмножество $M_{0} \subset M$, плотное в $M$. Следуюшая теорема является основной в работе.

TеоРема 5.1. Пусть непрерывное отображение $\Gamma: M \rightarrow d c b L_{1}$ удовлетворяет предположению (Н1). Тогда для любого әлемента $u(\cdot) \in C(M, \overline{\operatorname{dec}} \mathscr{F})$ и любой полунепрерывной снизу функции $\varphi: M \rightarrow(0,+\infty)$ найдется әлемент $v(\cdot) \in C(M, \overline{\operatorname{dec}} \mathscr{F})$ такой, что

$$
\begin{gathered}
\|u(\xi)-v(\xi)\|_{L_{1}}<\varphi(\xi), \quad \xi \in M, \\
v(\xi) \in \operatorname{ext} \Gamma(\xi), \quad \xi \in M_{0} .
\end{gathered}
$$

Если $M$ б-компактно, то включение (5.2) выполняется для всех $\xi \in M$.

Доказательству теоремы предпошлем ряд вспомогательных лемм.

Для непрерывного селектора $u(\cdot) \in C(M, \overline{\operatorname{dec}} \mathscr{F})$ и полунепрерывной снизу функции $\varphi: M \rightarrow(0,+\infty)$ обозначим через $\mathscr{H}_{u, \varphi}$ замыкание в топологии $C\left(M, L_{1}\right)$ множества всех непрерывных селекторов отображения $\overline{\operatorname{dec}} \mathscr{F}$, удовлетворяюших неравенству

$$
\|u(\xi)-v(\xi)\|_{L_{1}}<\frac{\varphi(\xi)}{2}, \quad \xi \in M
$$

которое является полным, замкнутым подмножеством пространства $C(M, \overline{\operatorname{dec}} \mathscr{F})$.

Возьмем фиксированные $\eta>0$ и $s \geqslant 1$. Положим

$$
\mathscr{H}_{\eta}^{s}=\left\{v(\cdot) \in \mathscr{H}_{u, \varphi} ; D^{s}(v(\xi))<\eta, \xi \in M_{0}\right\}
$$

ЛЕмма 5.1. Множсество $\mathscr{H}_{\eta}^{s}$ является $G_{\delta}$-подмножеством $\mathscr{H}_{u, \varphi}$. 
ДокАЗАТЕЛЬСтво. В соответствии с теоремой 4.1 множество $\mathscr{H}_{\eta}^{s}$ не пусто. Представим $M_{0}$ в виде $M_{0}=\bigcup_{n=1}^{\infty} M_{n}$, где $M_{n}, n \geqslant 1,-$ компактные подмножества из $M$. Положим

$$
\mathscr{H}_{\eta}^{s}(n)=\left\{v(\cdot) \in \mathscr{H}_{u, \varphi} ; D^{s}(v(\xi))<\eta, \xi \in M_{n}\right\}, \quad n \geqslant 1 .
$$

Тогда $\mathscr{H}_{\eta}^{s}=\bigcap_{n=1}^{\infty} \mathscr{H}_{\eta}^{s}(n)$. Поэтому для доказательства леммы достаточно показать, что множество $\mathscr{H}_{\eta}^{s}(n)$ открыто в $\mathscr{H}_{u, \varphi}$. Для этого рассмотрим множество $\mathscr{H}_{u, \varphi} \backslash \mathscr{H}_{\eta}^{s}(n), n \geqslant 1$, и покажем, что оно замкнуто в $\mathscr{H}_{u, \varphi}$.

Пусть $v(\cdot) \in \overline{\mathscr{H}_{u, \varphi} \backslash \mathscr{H}_{\eta}^{s}(n)}$, где черта вверху означает замњкание в топологии пространства $C\left(M, L_{1}\right)$. Тогда найдется последовательность $u_{k}(\cdot) \in$ $\mathscr{H}_{u, \varphi} \backslash \mathscr{H}_{\eta}^{s}(n), k \geqslant 1$, сходящаяся к $v(\cdot)$ в топологии пространства $C\left(M, L_{1}\right)$. Для каждого $u_{k}(\cdot), k \geqslant 1$, найдется точка $\xi_{k} \in M_{n}$ такая, что $D^{s}\left(u_{k}\left(\xi_{k}\right)\right) \geqslant \eta$, $k \geqslant 1$. Не нарушая общности, можно считать, что последовательность $\xi_{k} \in M_{n}$, $k \geqslant 1$, сходится к некоторому элементу $\xi \in M_{n}$. Так как последовательность $u_{k}\left(\xi_{k}\right), k \geqslant 1$, сходится к $v(\xi)$ в топологии пространства $L_{1}$, а функция $D^{s}(\cdot)$ в соответствии с леммой 3.2 полунепрерывна снизу, то $D^{s}(v(\xi)) \geqslant \eta$. Следовательно, $v(\cdot) \in \mathscr{H}_{u, \varphi} \backslash \mathscr{H}_{\eta}^{s}(n)$, и множество $\mathscr{H}_{\eta}^{s}(n)$ открыто в $\mathscr{H}_{u, \varphi}$. Поэтому $\mathscr{H}_{\eta}^{s}$ является $G_{\delta}$-подмножеством $\mathscr{H}_{u, \varphi}$. Лемма доказана.

Лемма 5.2. Мнохсество $\mathscr{H}_{\eta}^{s}$ плотно в $\mathscr{H}_{u, \varphi}$.

ДокаЗАТЕЛЬСтво. Возьмем произвольный элемент $v(\cdot) \in \mathscr{H}_{u, \varphi}$ и произвольное $\varepsilon>0$. Тогда найдется элемент $v_{1}(\cdot) \in C(M, \overline{\operatorname{dec}} \mathscr{F})$ такой, что

$$
\begin{aligned}
& \left\|u(\xi)-v_{1}(\xi)\right\|_{L_{1}}<\varphi(\xi) / 2, \\
& \left\|v(\xi)-v_{1}(\xi)\right\|_{L_{1}}<\varepsilon, \quad \xi \in M .
\end{aligned}
$$

Пусть

$$
d(\xi)=\min \left\{\varphi(\xi) / 2-\left\|u(\xi)-v_{1}(\xi)\right\|_{L_{1}}, \varepsilon-\left\|v(\xi)-v_{1}(\xi)\right\|_{L_{1}}\right\} .
$$

Функция $d(\xi)>0, \xi \in M$, и полунепрерьвна снизу. Тогда в соответствии с леммой $3.6[24]$ сушествует непрерьвная функция $c(\xi), 0<c(\xi)<d(\xi), \xi \in M$. Согласно теореме 4.1 существует непрерывньй селектор $w(\cdot)$ отображения $\overline{\operatorname{dec}} F$ такой, что

$$
\left\|v_{1}(\xi)-w(\xi)\right\|_{L_{1}}<c(\xi), \quad D^{s}(w(\xi))<\eta, \quad \xi \in M
$$

Тогда

$$
\begin{aligned}
\|u(\xi)-w(\xi)\|_{L_{1}} & \leqslant\left\|u(\xi)-v_{1}(\xi)\right\|_{L_{1}}+\left\|v_{1}(\xi)-w(\xi)\right\|_{L_{1}} \\
& \leqslant\left\|u(\xi)-v_{1}(\xi)\right\|_{L_{1}}+c(\xi) \\
& <\left\|u(\xi)-v_{1}(\xi)\right\|_{L_{1}}+\varphi(\xi) / 2-\left\|u(\xi)-v_{1}(\xi)\right\|_{L_{1}} \\
& \leqslant \varphi(\xi) / 2, \quad \xi \in M
\end{aligned}
$$

Аналогично получим, что

$$
\|v(\xi)-w(\xi)\|_{L_{1}}<\varepsilon, \quad \xi \in M
$$

Следовательно, $w(\cdot) \in \mathscr{H}_{\eta}^{s}$. Поскольку $\varepsilon>0$ произвольно, то множество $\mathscr{H}_{\eta}^{s}$ плотно в $\mathscr{H}_{u, \varphi}$. Лемма доказана. 
Лемма 5.3. $\mathscr{H}_{u, \varphi}=\overline{\left\{\bigcap_{n \geqslant 1} \bigcap_{s \geqslant 1} \mathscr{H}_{1 / n}^{s}\right\}}$, где черта означает замыкание в топологии пространства $C\left(T, L_{1}\right)$.

Лемма вытекает из лемм 5.1, 5.2 и теоремы Бэра о категориях [26].

Перейдем к доказательству теоремы 5.1.

ДокаЗАТЕЛЬСтво теоремы 5.1. Пусть $v(\cdot) \in\left\{\bigcap_{n \geqslant 1} \bigcap_{s \geqslant 1} \mathscr{H}_{1 / n}^{s}\right\}$. Тогда $D^{s}(v(\xi))<1 / n$ для всех $\xi \in M_{0}, s \geqslant 1$. Следовательно, $D^{s}(v(\xi))=0, \xi \in M_{0}$, $s \geqslant 1$. Тогда согласно лемме $3.2 v(\xi) \in \operatorname{ext} \Gamma(\xi), \xi \in M_{0}$. Поскольку $v(\cdot) \in \mathscr{H}_{u, \varphi}$, TO

$$
\|u(\xi)-v(\xi)\|_{L_{1}} \leqslant \varphi_{1}(\xi) / 2<\varphi_{1}(\xi), \quad \xi \in M
$$

Если $M \sigma$-компактно, то в качестве $M_{0}$ мы можем взять само пространство $M$. Теорема доказана.

ТЕОРема 5.2. Пусть непрерывное отображсение $\Gamma: M \rightarrow d c b L_{1}$ удовлетворяет предположсению $(\mathrm{H} 2)$. Тогда для любого әлемента $u(\cdot) \in C(M, \overline{\operatorname{dec}} \mathscr{F})$ и любой полунепрерывной снизу функиии $\varphi: M \rightarrow(0,+\infty)$ найдется әлемент $v(\cdot) \in C(M, \Gamma)$ такой, что выполняются неравенство (5.1) и включение (5.2). Если $M$ б-компактно, то включение (5.2) выполняется для всех $\xi \in M$.

Доказательство теоремы дословно повторяет доказательство теоремы 5.1, следует только в леммах 5.1-5.3 в качестве $\mathscr{H}_{u, \varphi}$ взять замыкание в топологии пространства $C\left(M, L_{1}\right)$ множества всех непрерывных селекторов отображения $\Gamma$, удовлетворяющих неравенству (5.3).

СЛЕДСТВИЕ 5.1. Пусть значениями отображения $Г: M \rightarrow d c b L_{1}$ являются множсества со свойством R-N. Тогда для любого непрерывного селектора $u(\cdot)$ отображения $\overline{\operatorname{dec}} \mathrm{st} \Gamma$ и любой полунепрерывной снизу функиии $\varphi: M \rightarrow(0,+\infty)$ существует непрерывный селектор $v(\cdot)$ отобрахсения $\overline{\mathrm{dec}} \mathrm{st} \Gamma$ такой, что выполняются неравенство (5.1), включение (5.2) и

$$
v(\xi) \in \overline{\operatorname{ext}} \Gamma(\xi), \quad \xi \in M \backslash M_{0}
$$

Если $M$ б-компактно, то включение (5.2) выполняется для всех $\xi \in M$.

Следствие вытекает из утверждения 4.1 и теоремы 5.1 , так как в качестве $\mathscr{F}$ мы можем взять отображение st $\Gamma$. Следует только заметить, что

$$
\overline{\operatorname{dec}} \operatorname{st} \Gamma(\xi) \subset \overline{\operatorname{ext}} \Gamma(\xi), \quad \xi \in M
$$

СЛЕДСТВИЕ 5.2. Пусть отображение $\Gamma: M \rightarrow d b L_{1}$ ограничено на $M u$ таково, что отображение $\overline{\mathrm{co}} \Gamma$ непрерывно и его значениями являются множсества со свойством R-N. Тогда существует непрерывный селектор и (·) отображения Г такой, что

$$
\begin{array}{ll}
u(\xi) \in \operatorname{ext} \overline{\operatorname{co}} \Gamma(\xi), & \xi \in M_{0}, \\
u(\xi) \in \overline{\operatorname{ext}} \overline{\operatorname{co}} \Gamma(\xi), & \xi \in M \backslash M_{0} .
\end{array}
$$


Еслик тому же $M$ б-компактно, то включение (5.5) выполняется для всех $\xi \in M$.

Так как отображение Г ограничено и его значениями являются разложимые множества, то отображение $\overline{\text { со }}$ о ограничено, и в соответствии с леммой 3.1 [4] значениями отображения со Г являются разложимые множества. Тогда согласно следствию 5.1 существует непрерывный селектор $u(\cdot)$ отображения $\overline{\mathrm{dec}} \mathrm{st} \overline{\mathrm{co}} \Gamma(\xi)$, такой, что выполняются включения (5.5), (5.6). Поскольку значениями отображения Г являются замкнутые множества, то st $\overline{c o} \Gamma(\xi) \subset \Gamma(\xi), \xi \in M[23]$, что в свою очередь влечет $\overline{\operatorname{dec}} \operatorname{st} \overline{c o} \Gamma(\xi) \subset \Gamma(\xi), \xi \in M$. Поэтому $u(\cdot)$ является искомым селектором отображения $Г$.

ЗАмЕчАНИЕ 5.1. Поскольку значениями отображения ехt Г являются в общем случае незамкнутые, невыпуклые множества, то теоремы 5.1, 5.2 и следствие 5.1 дают условия, при которых отображение ext $Г$ имеет непрерывный селектор.

ЗАмЕчАниЕ 5.2. В следствии 5.1 мы не предполагаем, что отображение Г обладает какими-либо свойствами непрерьвности или полунепрерьвности. Поэтому следствие 5.2 дает условия существования непрерывного селектора у многозначного отображения с замкнутыми разложимыми значениями, не обладающего свойствами непрерывности или полунепрерывности.

Перейдем теперь к примерам отображений $\Gamma: M \rightarrow d c b L_{1}$, широко используемых при изучении дифференциальных и эволюционных включений [2]-[6] и др.

Пусть $T$ - метрическое компактное пространство, $C(T, X)$ - пространство всех непрерывных отображений из $T$ в $X$ с топологией равномерной сходимости на $T$. При этих предположениях $C(T, X)$ является сепарабельным метрическим пространством.

Рассмотрим отображение типа Каратеодори $F: T \times X \rightarrow \operatorname{conv} X(\sigma)$, т.е. при каждом $x \in X$ отображение $F(T, X)$ измеримо по $t$ и при почти каждом $t$ непрерывно по $x$ в метрике Хаусдорфа $\operatorname{dist}(\cdot, \cdot)$.

Возьмем $M \subset C(T, X)$. Предположим, что отображение Г интегрально ограничено на $M$, т.е. существует суммируемая функция $\lambda: T \rightarrow \mathbb{R}^{+}$такая, что для любого $x(\cdot) \in M$ при почти всех $t$ выполняется неравенство

$$
\sup \{\|y\| ; y \in F(t, x(t))\} \leqslant \lambda(t) .
$$

Выберем плотное $\sigma$-компактное подмножество $M_{0} \subset M$.

УТВЕРЖ ДЕНИЕ 5.1. Пусть $F: T \times X \rightarrow \operatorname{conv} X(\sigma)$ - интегрально ограниченное на $M$ отображсение типа Каратеодори. Тогда существует непрерьвное отображсние $g: M \rightarrow L_{1}$ такое, что для каждого $x(\cdot) \in M_{0}$ при почти всех $t \in T$

$$
g(x)(t) \in \operatorname{ext} F(t, x(t))
$$

и для каждого $x(\cdot) \in M \backslash M_{0}$ при почти всех $t \in T$

$$
g(x)(t) \in \overline{\operatorname{ext}} F(t, x(t))
$$

Если $M$ б-компактно, то включение (5.7) выполняется для всех $x(\cdot) \in M$. 
ДокаЗАТЕЛЬСтво. Легко показать, что для каждого $x(\cdot) \in M$ отображение $F(t, x(t))$ измеримо. Обозначим через $S(F)(x(\cdot))$ совокупность всех интегрируемых селекторов отображения $F(t, x(t))$. Тогда $\Gamma(x(\cdot)) \in d \operatorname{conv} L_{1}(\sigma)$ [20], [22]. В силу леммы 3.1 отображение $\Gamma: M \rightarrow d \operatorname{conv} L_{1}(\sigma)$ непрерывно и ограничено. Поэтому согласно следствию 5.1 сушествует непрерывный селектор $g(\cdot)$ отображения $\overline{\operatorname{dec}} \operatorname{st} \overline{c o} \Gamma(\xi)$, такой, что выполняются включения (5.2), (5.4). Воспользовавшись следствием 3.1 , получаем, что отображение $g(\cdot)$ удовлетворяет включению (5.7).

Обозначим через $S(\overline{\mathrm{ext}} F)(x(\cdot))$ совокупность всех интегрируемых селекторов отображения $\overline{\operatorname{ext}} F(t, x(t))$. Легко показать, что оно является замкнутьм подмножеством в $L_{1}(T, X)$. Тогда $\overline{\operatorname{ext}} \Gamma(x(\cdot)) \subset S(\overline{\operatorname{ext}} F)(x(\cdot)), x(\cdot) \in M$. Воспользовавшись включением (5.4), получаем, что отображение $g(\cdot)$ удовлетворяет включению (5.8). Утверждение доказано.

УТВЕРЖДЕНИЕ 5.2. Пусть интегрально ограниченное многозначное отображение $F: T \times X \rightarrow X$ с замкнутыми, относительно слабо компактными значениями таково, что отображение $\overline{\mathrm{co}} F(t, x(t))$ типа Каратеодори. Предположим, что для каждого $x(\cdot) \in M$ отображение $F(t, x(t))$ измеримо. Тогда существует непрерьвное отображение $g: M \rightarrow L_{1}$ такое, что для каждого $x(\cdot) \in M$ при почти всех $t$

$$
g(x)(t) \in F(t, x(t))
$$

для каждого $x(\cdot) \in M_{0}$ при почти всех $t$

$$
g(x)(t) \in \operatorname{ext} \overline{c o} F(t, x(t)),
$$

для каждого $x(\cdot) \in M \backslash M_{0}$ при почти всех $t$

$$
g(x)(t) \in \overline{\operatorname{ext}} \overline{\operatorname{co}} F(t, x(t))
$$

ДокАЗАтЕЛЬСтво. Обозначим через $\mathscr{F}(x(\cdot))$ совокупность всех интегрируемых селекторов отображения $F(t, x(t))$, а через $\Gamma(x(\cdot))$ совокупность всех интегрируемых селекторов отображения $\overline{c o} F(t, x(t))$. Тогда $\mathscr{F}(x(\cdot)) \in d b L_{1}, \mathscr{F}(x(\cdot)) \subset$ $\Gamma(x(\cdot)), x(\cdot) \in M$, и отображение $\Gamma: M \rightarrow d \operatorname{conv} L_{1}(\sigma)$ непрерывно и интегрально ограничено на $M$. Согласно теореме $1.5[21] \overline{\mathrm{co}} \mathscr{F}(x(\cdot))=\Gamma(x(\cdot)), x(\cdot) \in M$. Тогда в соответствии со следствием 5.2 сушествует непрерывный селектор $g(\cdot)$ отображения $\mathscr{F}$, удовлетворяющий

$$
\begin{aligned}
& g(x(\cdot)) \in \operatorname{ext} \overline{\operatorname{co}} \mathscr{F}(x(\cdot)), \quad x(\cdot) \in M_{0}, \\
& g(x(\cdot)) \in \overline{\operatorname{ext}} \overline{\operatorname{co}} \mathscr{F}(x(\cdot)), \quad x(\cdot) \in M \backslash M_{0} .
\end{aligned}
$$

Воспользовавшись аргументами, использованными при доказательстве утверждения 5.1 , из (5.9), (5.10) получаем, что $g(x(\cdot))$ - искомое отображение. Утверждение доказано. 


\section{Список литературы}

1. Antosievicz A., Cellina A. Continuous selectors and differential relations // J. Differential Equations. 1975. V. 19. № 2. P. 386-398.

2. Fryszkowski A. Continuous selections for a class of nonconvex multivalued maps // Studia Math. 1983. V. 76. № 2. P. 163-174.

3. Bressan A., Colombo G. Extensions and selections of maps with decomposable values // Studia Math. 1988. V. 90. №1. P. 69-86.

4. Гончаров B. B., Толстоногов A. A. Совместные непрерьвные селекторы многозначных отображений с невыпуклыми значениями и их приложения // Матем. сб. 1991. Т. 182. № 7. C. 946-969.

5. Толстоногов A. А. Экстремальные селекторы многозначных отображений и принцип "бэнг-бэнг" для эволюционных включений // ДАН СССР. 1991. Т. 317. № 3. С. 589-593.

6. Гончаров B. B., Толстоногов A. A. Непрерьвные селекторы семейства невьпуклозначных отображений с некомпактной областью определения // Сиб. матем. журн. 1994. T. 35. №3. C. 537-553.

7. Cellina A. On the differential inclusion $\dot{x} \in[-1,1] / /$ Atti. Accad. Naz. Lincei Cl. Sci. Fis. Mat. Natur. Rend. Lincei (9) Math. Appl. 1980. V. 69. P. 1-6.

8. De Blasi F.S., Piangiani G. A Baire category approach to the existence of solutions of multivalued differential inclusions in Banach Spaces // Funkcial. Ekvac. 1982. V. 25. № 2. P. 153-162.

9. De Blasi F. S., Piangiani G. The Baire category method in existence problem for a class of multivalued differential equations with nonconvex right hand side // Funkcial. Ekvac. 1985. V. 28. № 2. P. 139-156.

10. De Blasi F. S., Piangiani G. Differential inclusions in Banach Spaces // J. Differential Equations. 1987. V. 66. P. 208-229.

11. De Blasi F. S., Piangiani G. Non-convex valued differential in Banach Spaces // J. Math. Anal. Appl. 1991. V. 157. P. 469-494.

12. De Blasi F. S., Piangiani G. On the density of extremal solutions of differential inclusions // Ann. Polon. Math. 1992. V. 56. № 2. P. 133-142.

13. Chuong P. V. Un resultat d'existence de solutions pour des equations differentielles multivoques // C. R. Acad. Sci. Paris Sér. I. Math. 1985. V. 30. P. 339-402.

14. Bressan A. Differential inclusions with non-closed non-convex right-hand side // Differential Integral Equations. 1990. V. 3. P. 633-638.

15. Суслов С. И. Нелинейньпй "бэнг-бэнг” принцип в $\mathbb{R}^{n} / /$ Матем. заметки. 1991. Т. 49. № 5. С. $110-116$.

16. Суслов С. И. Нелинейнпй "бэнг-бэнг” принцип в банаховом пространстве // Сиб. матем. журн. 1992. Т. 33. № 4. С. 142-154.

17. Бурбаки Н. Топологические векторные пространства. М.: ИЛ, 1959.

18. Эдвардс Р. Функциональньй анализ. Теория и приложения. М.: Мир, 1969.

19. Иоффе А. Д., Тихомиров В. М. Теория экстремальных задач. М.: Наука, 1974.

20. Himmelberg C. J. Measurable relations // Fund. Math. 1975. V. 87. № 1. P. 53-72.

21. Hiai F., Umegaki $H$. Integrals, conditional expectations and martingales of multivalued functions // J. Multivariate Anal. 1977. V. 7. P. 149-182.

22. Papageorgiou N.S. Representation of set-valued operators // Trans. Amer. Math. Soc. 1985. V. 292. № 2. P. 557-572.

23. Bourgin R. D. Geometric Aspects of Convex Sets with the Radon-Nikodym Property // Lect. Notes in Math. V. 993. Berlin, Heidelberg, New York, Tokyo: Springer-Verlag, 1983.

24. De Blasi F. S., Piangiani G. Remarks on Hausdorff continuous multifunctions and selections // Comment. Math. Univ. Carolin. 1983. V. 24. № 3. P. 553-561.

25. Келли Джс. Л. Общая топология. М.: Наука, 1981.

26. Куратовский K. Топология. Т. 1. М.: Мир, 1966.

Вычислительньй центр СО РАН

E-mail: aatol@icc.ccsoan.irkutsk.su
Поступила в редакцию 12.01 .1995 и 13.11 .1995 